\title{
Infrared Spectrum of Bromochlorofluoromethane
}

\author{
Earle K. Plyler and Mary A. Lamb
}

\begin{abstract}
The spectrum of bromochlorofluoromethane has been measured in order to compare the wave numbers of the fundamental bands with those of other substituted methanes containing the halogens. The positions of the absorption bands have been determined for the liquid and vapor states and all fundamentalbands except one at $220 \mathrm{~cm}^{-1}$ have been observed. The position of this band has been predicted from other bands that are classified as combinations of two fundamental bands, one of which was the $220 \mathrm{~cm}^{-1}$ band. Many of the bands of low or medium intensity have been classified as combinations or overtones of the nine fundamentals.
\end{abstract}

The spectra of many substituted methanes have been measured in the infrared, and normal coordinate calculations have been made on the fundamental modes of vibration of these molecules. ${ }^{1}$ Good agreement has been found between the experimental and theoretical values assigned to the fundamental vibrations.

The number of different fundamental vibrations can be determined from the symmetry of the molecule. For example, molecules like methane or tetrafluoromethane have four different vibrations, molecules of the type of chloroform and fluoroform have six different fundamental frequencies, and molecules of the type of methylene chloride and bromochlorofluoromethane have nine separate fundamental frequencies. When the hydrogens of methane are substituted by the different halogen atoms, the number of molecular constants increases, and it is more difficult to obtain good agreement between the observed and calculated positions for the bands. Methods for carrying out the calculations with some simplifications have been described by Decius. ${ }^{2}$ The compound bromochlorofluoromethane is of interest for it does not have a center or axis of symmetry.

The purpose of this investigation was to study the spectrum of bromochlorofluoromethane and to assign the fundamental bands. A comparison of the spectrum of bromochlorofluoromethane with the assigned vibrations in the spectra of related halogensubstituted methanes is helpful in making the assignments and in attributing them to motions of particular atoms. The dichlorofluoromethane assignments are also helpful in making the identification of the $\mathrm{C}-\mathrm{Cl}$ and $\mathrm{C}-\mathrm{F}$ bending and stretching vibrations in bromochlorofluoromethane. The identification of the $\mathrm{C}-\mathrm{Br}$ bending and stretching vibrations were made by comparison of the spectrum with the assigned spectrum of dibromochloromethane.

The bromochlorofluoromethane was obtained from Halogen Chemicals Inc., where it was repurified so that it was suitable for spectroscopic measurements. The compound was redistilled into three fractions by W. Harold Smith, of the Bureau's Organic Chemistry Section. Tests of the purity of two of the fractions were made in the Mass Spectrometry Section of

1 G. Herzberg, Infrared and Raman spectra of polyatomic molecules (D. Van Nostrand Co., Inc., New York, N. Y., 1945)

2 J. C. Decius, J. Chem. Phys, 16, 214 (1948). this Bureau, and the results showed that the distilled fraction with the intermediate boiling point, $37^{\circ} \mathrm{C}$, was 98 percent of bromochlorofluoromethane. The principal impurities were probably dichlorobromomethane and dichlorofluoromethane. The fraction with the lower boiling point, $29.5^{\circ}$ to $35.8^{\circ}$ C, showed 95.1 percent of bromochlorofluoromethane, and the most likely impurities were dibromofluoromethane, dichlorofluoromethane, and dichlorobromomethane. The spectra of the three fractions were essentially the same. Some slight differences in the intensity of weak bands were noted; these coincided in position with the strongest bands in the spectra of the most likely impurities and are attributed to those molecules. These are discussed later.

The infrared absorption spectrum of bromochlorofluoromethane has been measured in both the liquid and vapor states. Although all three distillation fractions were measured, only the spectrum of the fraction with the intermediate boiling point, $37^{\circ} \mathrm{C}$, will be presented since this was the fraction of highest purity. In figure $1, \mathrm{~A}$, is shown the region from 2 to $15 \mu$ of the spectrum of the compound in the liquid state as measured by a Perkin-Elmer spectrometer with the lithium fluoride and sodium chloride prisms. The broken line portions of the curve represent the weak bands, which are probably due to the impurities present in the fraction, and they also represent regions of atmospheric water absorption where the shapes and positions of the bands were difficult to determine accurately. These regions have been plotted using the spectrum measured on a Baird infrared spectrophotometer. Five of the nine fundamental vibrations are present in this region, and most of the other bands have been assigned as combination or overtone bands. A list of the bands with their assignments is included in table 1 . The region from 15 to $23 \mu$, as measured with a potassium bromide prism, and the region from 23 to $38 \mu$, as measured with a thallium bromide-iodide prism, are shown in figure 1, B. Three of the remaining four fundamental bands have been assigned to bands in this region. The ninth fundamental vibration has not been observed directly, but may be a value of $220 \mathrm{~cm}^{-1}$ on the basis of observed combination and difference bands. This band is beyond the range of the thallium bromide-iodide prism. 
In figure 1, C, are shown the more intense bands in the vapor spectrum in the region from 2 to $23 \mu$. Most of these bands show evidence of $\mathrm{P}, \mathrm{Q}$, and $\mathrm{R}$ branches. The $\mathrm{C}-\mathrm{H}$ stretching vibration at 3,023 $\mathrm{cm}^{-1}$ is shown on an enlarged scale so that the detail of the band can be seen more clearly. The bands shown are of the same relative intensities as they were in the measurements of the liquid state. There is noted, however, a slight shift to larger wave numbers of not more than $10 \mathrm{~cm}^{-1}$ for the vapor state from the wave numbers observed in the liquid state spectrum.

In table 1 are listed all of the observed bands as observed in the liquid state of appreciable intensity with their intensities denoted by abbreviations for very weak, weak, medium, strong, or very strong. The assignments of the bands have also been given as fundamental, combination, overtone, or difference bands. All of the intense bands are classified and fall into a consistent array based on the nine fundamental vibrations. Several of the assignments for weaker bands may not be correct, and eight observed weak bands have not been assigned in the table. It is possible that these weak bands arise from the small quantities of impurities in the compound. For example, the bands observed at 605,721 , and 1,168 $\mathrm{cm}^{-1}$ may be due to the dichlorobromomethane fundamental frequencies $\nu_{3}, \nu_{7}$, and $\nu_{6}$ respectively, also the observed band at $848 \mathrm{~cm}^{-1}$ may arise from trichlorofluoromethane. The unassigned band at 790 $\mathrm{cm}^{-1}$ possibly may be explained as a combination band of dichlorobromomethane, $\nu_{3}+\nu_{5}$. To an ap- proximation the bands may be considered as arising from stretching and bending vibrations of each peripheral atom $(\mathrm{H}, \mathrm{Br}, \mathrm{Cl}, \mathrm{F})$ with respect to the carbon atom. This would account for eight fundamental modes of vibration. The ninth fundamental band could be considered as a torsional motion of the molecule, but since the hydrogen atom is so much lighter than the halogens, the motion in this mode may equally well be considered a second degree of freedom of bending of the $\mathrm{CH}$ bond.

The spectrum of bromochlorofluoromethane should be similar to that of dichlorofluoromethane except for the modes of motion primarily involving the C-Br group, which should occur at lower frequencies for bromochlorofluoromethane than the corresponding $\mathrm{C}-\mathrm{Cl}$ group in dichlorofluoromethane. Thus, for example, the band with the wave number of $3,023 \mathrm{~cm}^{-1}$ is identified as the $\mathrm{C}-\mathrm{H}$ stretching, $\nu_{1}$, vibration and the bands with wave numbers of 1,202 and $1,299 \mathrm{~cm}^{-1}$ are classified as the two bending vibrations of $\mathrm{CH}$. These two types of vibrations are referred to as $\nu^{\mathrm{CH}}$ and $\delta^{\mathrm{CH}}$, respectively, in table 1 , and the same type of symbols are used to designate the other bands. Similar relationships hold between the unsymmetrical $\mathrm{CHBrClF}$ and the other kindred methane derivatives.

A comparison of the fundamentals of all four related molecules is shown in table 2. The assignments of the observed bands of the three latter methanes have been made and will be published later with a group of substituted methane derivatives.
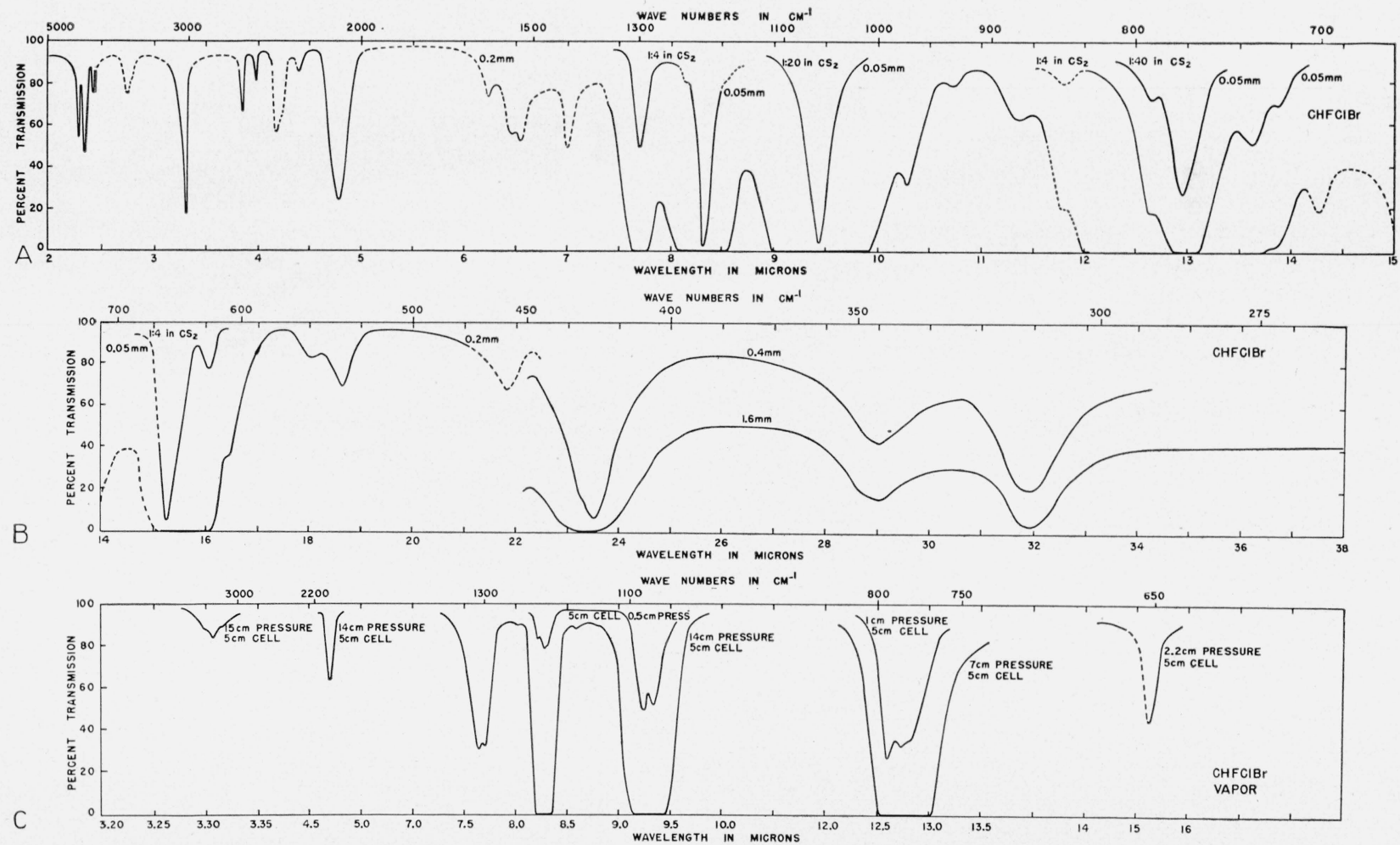

FIGURE 1. Infrared spectrum of bromochlorofluoromethane.

A, Liquid state from 2 to $15 \mu$; B, liquid state from 14 to $38 \mu$; C, intense bands, vapor state from 3 to $16 \mu$. 
TABLE 1. Classification of the observed bands of bromochlorofluoromethane

\begin{tabular}{|c|c|c|c|c|}
\hline Type & $\begin{array}{l}\text { Assign. } \\
\text { ment }\end{array}$ & Intensity & $\begin{array}{l}\text { Wave- } \\
\text { length }\end{array}$ & $\begin{array}{c}\text { Wave } \\
\text { number }\end{array}$ \\
\hline $\begin{array}{l}\delta_{\mathrm{CBr}} \\
\delta \mathrm{CCl} \\
\delta^{\mathrm{CF}}\end{array}$ & $\begin{array}{l}\nu_{2} \\
\nu_{5} \\
\nu_{3}-\nu_{5} \\
\nu_{4} \\
\nu_{3}-\nu_{2}\end{array}$ & $\begin{array}{l}\mathrm{W} \\
\mathrm{VW} \\
\mathrm{M} \\
\mathrm{W}\end{array}$ & $\begin{array}{c}\boldsymbol{\mu} \\
45.5 \\
31.9 \\
29.0 \\
23.5 \\
23.3\end{array}$ & $\begin{array}{r}c m^{-1} \\
\text { a } 220 \\
313 \\
345 \\
426 \\
429\end{array}$ \\
\hline & $\begin{array}{l}\nu_{7}-\nu_{5} \\
\nu_{2}+\imath_{5} \\
\nu_{7}-\nu_{2}\end{array}$ & $\begin{array}{c}W \\
W \\
\text { VW } \\
W \\
M\end{array}$ & $\begin{array}{l}21.83 \\
18.64 \\
18.05 \\
16.78 \\
16.53\end{array}$ & $\begin{array}{l}458 \\
536 \\
554 \\
596 \\
605\end{array}$ \\
\hline $\begin{array}{l}{ }_{\nu} \mathrm{CBr} \\
{ }_{\nu} \mathrm{CCl}\end{array}$ & $\begin{array}{l}2 \nu_{5} \\
\nu_{3} \\
\\
\nu_{4}+\nu_{5} \\
\nu_{7}\end{array}$ & $\begin{array}{c}M \\
\text { VS } \\
W \\
M \\
\text { VS }\end{array}$ & $\begin{array}{l}16.07 \\
15.26 \\
13.87 \\
13.62 \\
12.95\end{array}$ & $\begin{array}{l}622 \\
655 \\
721 \\
734 \\
772\end{array}$ \\
\hline & $\begin{array}{l}2 \nu_{4} \\
\nu_{3}+\nu_{2} \\
3 \nu_{5} \\
\nu_{3}+\nu_{5}\end{array}$ & $\begin{array}{r}\mathrm{M} \\
\mathrm{W} \\
\mathrm{W} \\
\mathrm{VVW} \\
\mathrm{W}\end{array}$ & $\begin{array}{l}12.65 \\
11.79 \\
11.37 \\
10.73 \\
10.29\end{array}$ & $\begin{array}{l}790 \\
848 \\
880 \\
932 \\
972\end{array}$ \\
\hline $\begin{array}{l}\nu_{\mathrm{CF}} \\
\delta^{\mathrm{CH}} \\
\delta^{\mathrm{CH}}\end{array}$ & $\begin{array}{c}\nu_{9} \\
2 \nu_{4}-\nu_{5} \\
\nu_{8} \\
\nu_{6}\end{array}$ & $\begin{array}{r}\mathrm{VS} \\
\mathrm{VW} \\
\mathrm{VS} \\
\mathrm{VW} \\
\mathrm{S}\end{array}$ & $\begin{array}{l}9.43 \\
8.56 \\
8.32 \\
8.04 \\
7.69\end{array}$ & $\begin{array}{l}1060 \\
1168 \\
1202 \\
1243 \\
1299\end{array}$ \\
\hline & $\begin{array}{l}\nu_{3}+\nu_{7} \\
2 \nu_{7} \\
\nu_{5}+\nu_{6} \\
2 \nu_{9}\end{array}$ & $\begin{array}{r}W \\
W \\
V W \\
V V W \\
M\end{array}$ & $\begin{array}{l}7.0 \\
6.5 \\
6.4 \\
6.2 \\
4.75\end{array}$ & $\begin{array}{r}\text { b } 1429 \\
\text { b } 1538 \\
\text { b } 1563 \\
\text { b } 1613 \\
2105\end{array}$ \\
\hline 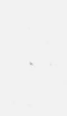 & $\begin{array}{l}2 \nu_{8} \\
2 \nu_{9}+\nu_{5} \\
\nu_{8}+\nu_{6} \\
2 \nu^{\prime} 6\end{array}$ & $\begin{array}{c}\text { VW } \\
V W \\
W \\
V W \\
W\end{array}$ & $\begin{array}{l}\text { 4. } 40 \\
4.182 \\
4.132 \\
3.979 \\
3.847\end{array}$ & $\begin{array}{l}2273 \\
2391 \\
2420 \\
2513 \\
2599\end{array}$ \\
\hline${ }_{\nu} \mathrm{CH}$ & $\begin{array}{l}\nu_{1} \\
\nu_{1}+\nu_{3} \\
\nu_{1}+\nu_{9} \\
\nu_{1}+\nu_{8} \\
\nu_{1}+\nu_{6}\end{array}$ & $\begin{array}{c}\mathrm{M} \\
\mathrm{VW} \\
\mathrm{VW} \\
\mathrm{W} \\
\mathrm{W}\end{array}$ & $\begin{array}{l}3.308 \\
2.75 \\
2.426 \\
2.348 \\
2.290\end{array}$ & $\begin{array}{l}3023 \\
3636 \\
4122 \\
4259 \\
4367\end{array}$ \\
\hline
\end{tabular}

a Predicted value

b Value from Baird spectrophotometer.
The bands of bromofluorochloromethane have slightly smaller wave numbers than those of the corresponding dichlorofluoromethane bands. However the $\mathrm{C}-\mathrm{H}$ stretching vibration is about equal for both compounds.

TABLE 2. Comparison of the fundamental frequencies of dichlorofluoromethane, bromochlorofluoromethane, bromodichlorofluoromethane, and dibromochloromethane

\begin{tabular}{|c|c|c|c|c|}
\hline \multirow{2}{*}{$\begin{array}{c}\text { Assign- } \\
\text { ment }\end{array}$} & \multicolumn{4}{|c|}{ Wave number $\left(\mathrm{cm}^{-1}\right)$} \\
\hline & $\mathrm{CHCl}_{2} \mathrm{~F}$ & $\mathrm{CHBrClF}$ & $\mathrm{CHBrCl}_{2}$ & $\mathrm{CHBr}{ }_{2} \mathrm{Cl}$ \\
\hline$\nu_{2}$ & 276 & 220 & 220 & 168 \\
\hline$\nu_{5}$ & 368 & 313 & 215 & 201 \\
\hline$\nu_{4}$ & 458 & 426 & 330 & 278 \\
\hline$\nu_{3}$ & 742 & 655 & 604 & 570 \\
\hline$\nu 7$ & 804 & 772 & 719 & 746 \\
\hline$\nu_{9}$ & 1079 & 1060 & 760 & 657 \\
\hline$\nu_{8}$ & 1242 & $\begin{array}{l}1202 \\
\end{array}$ & 1211 & 1144 \\
\hline$\nu_{6}$ & 1304 & 1299 & 1168 & 1189 \\
\hline$\nu_{1}$ & 3023 & 3023 & 3040 & 3034 \\
\hline
\end{tabular}

The Raman spectrum of bromochlorofluoromethane has been measured by Glockler and Leader. ${ }^{3}$ They found Raman lines that check well in wave number with the fundamentals assigned in the present work. Except for the CH stretching vibration the Raman values were not assigned to different types of vibrations of the molecules.

${ }^{3}$ G. Glockler and G. R. Leader, J. Chem Phys 8, 699 (1940).

Washington, October 23, 1950 